\title{
Myelofibrosis Transformation in Essential Thrombocythemia
}

National Cancer Institute

\section{Source}

National Cancer Institute. Myelofibrosis Transformation in Essential Thrombocythemia. NCl Thesaurus. Code C126806.

Myelofibrosis that develops in a patient with history of essential thrombocythemia. 